\title{
An Influence of the Wall Acoustic Impedance on the Room Acoustics. The Exact Solution
}

\author{
Adam BRAŃSKI, Anna KOCAN-KRAWCZYK, Edyta PRĘDKA \\ The Faculty of Electrical and Computer Engineering \\ Department of Acoustics \\ Rzeszów University of Technology \\ Powstańców Warszawy 12, 35-959 Rzeszów, Poland; e-mail: abranski@prz.rzeszow.pl
}

(received March 17, 2017; accepted June 22, 2017)

\begin{abstract}
The Fourier method is applied to the description of the room acoustics field with the combination of uniform impedance boundary conditions imposed on some walls. These acoustic boundary conditions are expressed by absorption coefficient values In this problem, the Fourier method is derived as the combination of three one-dimensional Sturm-Liouville (S-L) problems with Robin-Robin boundary conditions at the first and second dimension and Robin-Neumann ones at the third dimension. The Fourier method requires an evaluation of eigenvalues and eigenfunctions of the Helmholtz equation, via the solution of the eigenvalue equation, in all directions. The graphic-analytical method is adopted to solve it It is assumed that the acoustic force constitutes a monopole source and finally the forced acoustic field is calculated. As a novelty, it is demonstrated that the Fourier method provides a useful and efficient approach for a room acoustics with different values of wall impedances. Theoretical considerations are illustrated for rectangular cross-section of the room with particular ratio. Results obtained in the paper will be a point of reference to the numerical calculations.
\end{abstract}

Keywords: Fourier analysis; architectural acoustics; absorption coefficients; boundary-value problems.

\section{Introduction}

The influence of wall impedances on the acoustic field in the cinema, theatre room, and so on is an important and interesting problem both from the practical and theoretical points of view. Thus, there is a need to apply some methods for solving the acoustic room problem and walls with arbitrary impedances. But the description of the sound field in such a room is not simple. For this purpose several approximate and exact methods have been developed; most of these methods in general forms are described in (MORSE, INGARD, 1987; BRAŃSKI, 2013).

Approximate methods, based on heuristic premises, are used in many papers. They use statistical-acoustic methods (SUMMERS, 2012), the diffusion-equation model (LUIZARD et al., 2014), geometric acoustics methods (LuizARD et al., 2014; LEHMAnN, JohANsSON, 2008), the combination of radiosity method, geometrical acoustic one (KORANY et al., 2001), and so on.

Wave-based approximate methods solve the wave equation in an approximate manner. In the descrip- tion of the sound field, the most useful methods are finite element method (FEM) (OKUZONO et al., 2014; Thompson, Pinsky, 1995), the singular boundary element method (BEM), (LIN et al., 2014; Fu et al., 2014; CHEN et al., 2014), the nonsingular BEM (BRAŃsKI et al., 2012; BrAŃsKI, BorkowsKA, 2015) and the finite difference method (FDM) (LopEz et al., 2013). All the above methods are numerical.

The Fourier method belongs to exact ones and it may be used for solving boundary problems of the room acoustics (BLACKSTOCK, 2000; KUTTRUFF, 2000), being inherent to the modal analysis (NAKA et al., 2005). It requires an evaluation of eigenvalues of the Helmholtz equation assuming some boundary conditions imposed on the walls. Since the acoustic eigenvalue equation is complicated, the numerical method should be applied to find eigenvalues (NAKA et al., 2005; KuttrufF, 2000; Bistafa, Morrissey, 2003), e.g. the Newton or bisection method. Describing the wave nature of the sound field (MEISSNER, 2009) and the modal localisation is the benefit of the Fourier method (MEIssneR, 2009). Other versions of 
this method may be found in (XU, SommerfeldT, 2010). The Fourier method is difficult to apply for rooms with complex shapes and complex boundary conditions, and in more practical cases it is unusable (MEIssner, 2012; 2013a; 2013b).

Although the modal theory of the room acoustics was fully established many years ago, the solution of it is still incomplete. In particular, there is no exact general method for finding eigenvalues and, consequently, room modes for walls with arbitrary impedances. Only for rooms with perfectly flexible or rigid walls this problem is exactly solved (BLACKSTOCK, 2000; KUTTRUFF, 2000). But for some impedance on each pair of parallel walls it is only estimated.

To make a more general analysis of the modal acoustic boundary problem, it is appropriate to refer to the Sturm-Liouville eigenvalue problem (JOHNSON, 2006; Dautray, Lions, 2000; Korn, Korn, 1968). The S-L problems arise throughout applied mathematics and in a standard form they describe the vibrational modes of various systems, among others the acoustic ones. Then, they arise directly as eigenvalue problems in one-dimensional space (1D). However, they also commonly arise from linear PDEs in multidimensional spaces when the equations are separable in some coordinate systems, exactly like in the three dimensional acoustic problem.

The aim of the paper is to derive an exact solution of the 3D real acoustic problem by the Fourier method. The arbitrary but uniform impedances are imposed on separate walls. The values of the wall impedance, for all possible values of wall absorption coefficients, are considered. Assuming different absorption coefficients imposed on walls, the appropriate eigenvalue equations are solved graphically, hence the set of eigenvalues and consequently the set of modes is obtained. Next, the forced acoustic field is calculated. The theory is verified via several numerical calculations.

\section{Regular S-L problem}

The regular S-L problem is defined in (KASHDAN, 2017; FASSHAUER, 2011); some part of the theory, useful for the $3 \mathrm{D}$ acoustic problem, is repeated below in 1D space. Consider the S-L differential equation,

$$
\begin{array}{r}
D_{x}\left(R(x) D_{x} X(x)\right)+(Q(x)+\lambda P(x)) X(x)=0, \\
x=\left(a_{x}, b_{x}\right) \equiv \Omega,
\end{array}
$$

with the Robin boundary conditions (FASSHAUER, 2011; PEIRCE, 2014):

$$
\begin{gathered}
A_{1 a} X\left(a_{x}\right)+A_{2 a} D_{x} X\left(a_{x}\right)=0, \\
A_{1 b} X\left(b_{x}\right)+A_{2 b} D_{x} X\left(b_{x}\right)=0,
\end{gathered}
$$

where $a_{x}, b_{x}$ are boundary points of the $x$-coordinate in Cartesian coordinates; $D_{x}(\ldots)=\mathrm{d}(\ldots) / \mathrm{d} x, R(x)$, and
$Q(x)$ are coefficient functions, $P(x)$ is the weighting function, $R(x)$ and $P(x)$ are continuous on $\left[a_{x}, b_{x}\right]$ and $Q(x)$ is continuous on $\left(a_{x}, b_{x}\right), \lambda$ is an eigenvalue.

Note that if $R(x)=1, Q(x)=0$, and $P(x)=1$, the S-L equation leads to the 1D Helmholtz equation. It is proved that all conclusions derived for S-L problem are valid for 1D Helmholtz problem and they are also valid for 3D Helmholtz one. Furthermore, 3D boundary problem for the Helmholtz equation with Robin conditions is a product of three 1D problems for Helmholtz problem in separate coordinates.

\section{Three dimensional acoustic problem}

Let the 3D acoustic boundary problem in the cuboid, physical domain $\Omega$ with the boundary $\Gamma$ of this domain be given. The mathematical model is described by the $3 \mathrm{D}$ wave equation and Robin boundary conditions.

$$
\begin{aligned}
D^{2} u(\mathbf{x}, t)-\left(1 / c^{2}\right) D_{t}^{2} u(\mathbf{x}, t) & =f(\mathbf{x}, t), & & \mathbf{x} \in \Omega, \\
A_{1} u(\mathbf{x}, t)+A_{2} D_{\mathbf{n}} u(\mathbf{x}, t) & =0, & & \mathbf{x} \in \Gamma,
\end{aligned}
$$

where the initial conditions are assumed equal to zeros, $c[\mathrm{~m} / \mathrm{s}]$ is the speed of sound in that medium; $\mathbf{x}=(x, y, z)[\mathrm{m}] ; t[\mathrm{~s}]$ is the time; $u=u(\mathbf{x}, t)$, acoustic potential as a function of time and geometrical variables; $f(\mathbf{x}, t)$ is the exciting of the acoustic wave in $3 \mathrm{D}$, it is the harmonic monopole acoustic source which may be constituted by the sphere with a very little radius; $A_{1}, A_{2}$ are arbitrary constants, and $\mathbf{n}$ is a unit normal vector to the $\Gamma$ pointing outward $\Omega, D^{2} u(\mathbf{x}, t)=$ $\frac{\partial^{2} u}{\partial x^{2}}+\frac{\partial^{2} u}{\partial y^{2}}, D_{t}^{2} u(\mathbf{x}, t)=\frac{\partial^{2} u}{\partial t^{2}}, D_{\mathbf{n}} u(\mathbf{x}, t)=\frac{\partial u}{\partial n}$.

\subsection{Separation of variables}

Using Fourier method, first it is assumed that (NETA, 2012; Bowles, 2007)

$$
u(\mathbf{x}, t)=U(\mathbf{x}) T(t),
$$

and next

$$
U(\mathbf{x})=X(x) Y(y) Z(z),
$$

where $U(\mathbf{x})$ is the acoustic potential as a function of spatial variables only.

As it can be seen, substituting Eqs. (6) and (7) into Eq. (4) yields four ordinary differential equations (ODE) expressing $T(t), X(x), Y(y)$, and $Z(z)$. Combining the last three ODEs yields a partial differential equation (PDE) for $U(\mathbf{x})$. So, the wave Eq. (4) generates four ODEs:

$$
\begin{aligned}
D_{t}^{2} T(t)+\omega^{2} T(t) & =0, & & \\
D_{x}^{2} X(x)+k_{x}^{2} X(x) & =0, & & x \in\left(a_{x}, b_{x}\right), \\
D_{y}^{2} Y(y)+k_{y}^{2} Y(y) & =0, & & y \in\left(a_{y}, b_{y}\right), \\
D_{z}^{2} Z(z)+k_{z}^{2} Z(z) & =0, & & z \in\left(a_{z}, b_{z}\right),
\end{aligned}
$$


where $\omega[\mathrm{rad} / \mathrm{s}]$ is the angular frequency; $k[\mathrm{rad} / \mathrm{m}]$ is the wave number: $k=\omega / c ; k^{2}=k_{x}^{2}+k_{y}^{2}+k_{z}^{2}$.

\subsection{Acoustic boundary conditions}

Equations (8)-(11) are derived from Eq. (4) and together with the boundary condition, Eq. (5), constitute a new formulation of the boundary problem. To make it the acoustic boundary problem, the boundary condition Eq. (5) ought to be interpreted on the basis of acoustics.

First of all, note that the function $T(t)=\exp (i \omega t)$, where $i=\sqrt{-1}, \omega$ is the angular frequency, satisfies Eq. (8). Substituting this function to the boundary condition Eq. (5) leads to

$$
A_{1} U(\mathbf{x})+A_{2} D_{\mathbf{n}} U(\mathbf{x})=0, \quad \mathbf{x} \in \Gamma .
$$

In acoustics, the Robin boundary condition corresponds to specifying the surface acoustic impedance,

$$
z(\mathbf{x})=p(\mathbf{x}) / v(\mathbf{x}),
$$

where $p(\mathbf{x})[\mathrm{Pa}]$ is the acoustics pressure; $v(\mathbf{x})[\mathrm{m} / \mathrm{s}]$ is the particle velocity. forms

Sound parameters described with $U(\mathbf{x})$ take the

$$
\begin{aligned}
& p(\mathbf{x})=i \rho \omega U(\mathbf{x}), \\
& v(\mathbf{x})=-D_{\mathbf{n}} U(\mathbf{x})=-\operatorname{grad} U(\mathbf{x}),
\end{aligned}
$$

where $\rho\left[\mathrm{kg} / \mathrm{m}^{3}\right]$ is the air density.

As it can be seen, the impedance boundary condition, Eq. (13), can be written in the form

$$
z(\mathbf{x}) D_{\mathbf{n}} U(\mathbf{x})+i \rho \omega U(\mathbf{x})=0, \quad \mathbf{x} \in \Gamma .
$$

Hereunder this boundary condition is considered; note that comparing Eq. (16) to Eq. (12) one obtains $A_{1}=\rho \omega$ and $A_{2}=z(\mathbf{x})$.

In practice, the acoustic impedance $z(\mathbf{x})\left[\mathrm{N} \cdot \mathrm{s} / \mathrm{m}^{3}\right]$ is in fact the acoustic impedance of any material and it is wined through the measure of the absorption coefficient $\alpha(\mathbf{x})$. There are several methods to measure the $\alpha(\mathbf{x})$ or the acoustic impedance of acoustic materials. The classification of them is given in (GERAI, 1993) and three main groups of methods are specified there: reverberation room methods (ISO 354, 2003; Ducourneaua, Planeaub, 2003), in situ reflection methods (TAKAHASHI, 2005), and tube methods (ISO 10354-1, 1996). Both $\alpha(\mathbf{x})$ and $z(\mathbf{x})$ are connected to each other by the formula (KUTTRUFF, 2000)

$$
z(\mathbf{x})=\rho c \frac{1+(1-\alpha(\mathbf{x}))^{1 / 2}}{1-(1-\alpha(\mathbf{x}))^{1 / 2}} .
$$

For complex impedance, the boundary conditions will typically depend on frequency and consequently, both the eigenfunctions and resonant frequencies depend on frequency.

On account of Eq. (7), instead of Eq. (15), it is

$$
\begin{gathered}
D_{\mathbf{n}} X(x)+z_{0}(x) X(x)=0, \quad x \in\left(a_{x}, b_{x}\right), \\
D_{\mathbf{n}} Y(y)+z_{0}(y) Y(y)=0, \quad y \in\left(a_{y}, b_{y}\right), \\
D_{\mathbf{n}} Z(z)+z_{0}(z) Z(z)=0, \quad z \in\left(a_{z}, b_{z}\right),
\end{gathered}
$$

where $z_{0}(\mathbf{x})=(\omega \rho) / z(\mathbf{x})$.

\subsection{Solution of the acoustic modal problem}

For the practical acoustic case the floor is modelled through the rigid boundary condition (Neumann/ $N$ ), but walls and ceiling are modelled through impedance boundary conditions (Robin/ $R$ ).

Further on the following is assumed (see also Fig. 1):

- in the $x$-direction, impedance boundary conditions Eq. (18), denoted by $R$, namely

$$
\begin{aligned}
& D_{\mathbf{n}} X\left(a_{x}\right)+z_{0}\left(a_{x}\right) X\left(a_{x}\right)=0, \quad D_{\mathbf{n}}(\ldots)=-D_{x}(\ldots), \\
& D_{\mathbf{n}} X\left(b_{x}\right)+z_{0}\left(b_{x}\right) X\left(b_{x}\right)=0, \quad D_{\mathbf{n}}(\ldots)=D_{x}(\ldots),
\end{aligned}
$$

- in the $y$-direction, impedance boundary conditions Eq. (19), denoted by $R$, namely

$$
\begin{gathered}
D_{\mathbf{n}} Y\left(a_{y}\right)+z_{0}\left(a_{y}\right) Y\left(a_{y}\right)=0, \quad D_{\mathbf{n}}(\ldots)=-D_{y}(\ldots), \\
D_{\mathbf{n}} Y\left(b_{y}\right)+z_{0}\left(b_{y}\right) Y\left(b_{y}\right)=0, \quad D_{\mathbf{n}}(\ldots)=D_{y}(\ldots),
\end{gathered}
$$

- in the $z$-direction, the rigid boundary condition at $a_{y}$, denoted by $N$, and the impedance boundary condition at $b_{y}$, denoted by $R$, hence from Eq. (20) it holds that

$$
\begin{aligned}
D_{\mathbf{n}} Z\left(a_{z}\right) & =0, \quad D_{\mathbf{n}}(\ldots)=-D_{z}(\ldots), \\
D_{\mathbf{n}} Z\left(b_{z}\right)+z_{0}\left(b_{z}\right) Z\left(b_{z}\right) & =0, \quad D_{\mathbf{n}}(\ldots)=D_{z}(\ldots) .
\end{aligned}
$$

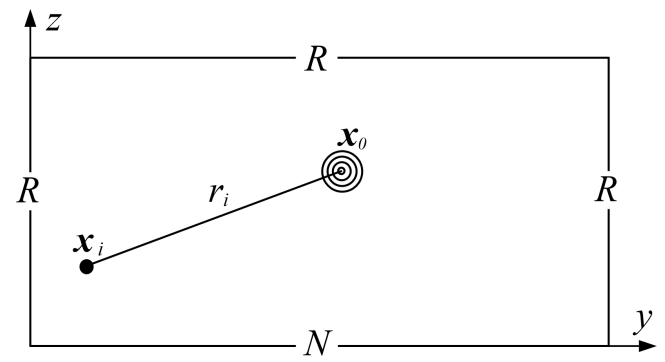

Fig. 1. Cross-section geometry of the problem; $x_{0}$ - source point, $x_{i}$ - arbitrary domain point, $r_{i}$ - distance between $x_{0}$ and $x_{i}, R$ - Robin boundary condition, $N-$ Neumann boundary condition. 
Hereunder eigenvalues and eigenfunctions in the $x$-direction and $y$-direction are derived in the case when the impedance at the left end $z_{0}\left(a_{x}\right) / z_{0}\left(a_{y}\right)$ is different from the impedance at the right end $z_{0}\left(b_{x}\right) / z_{0}\left(b_{y}\right)$. Thus, it is necessary to solve Eq. (9) and Eq. (10) with impedance boundary conditions Eqs. (21) and Eq. (22); the solution of Eq. (9) is presented in details, but the solution of Eq. (10) is written by analogy. form

The general solution of Eq. (9) is assumed in the

$$
X(x)=C_{1} \cos \left(k_{\mu} x\right)+C_{2} \sin \left(k_{\mu} x\right),
$$

hereunder $k_{\mu}=k_{x}$.

Substituting Eq. (27) into Eq. (21) and Eq. (22) gives $C_{1}=\left(C_{2} k_{x}\right) / z_{0}\left(a_{x}\right)$ and after some calculations one obtains the eigenvalues equation

$$
\tan \left(w_{x}\right)=\frac{w_{x}\left(Z_{a x}+Z_{b x}\right)}{w_{x}^{2}-Z_{a x} Z_{b x}},
$$

where $w_{x}=k_{\mu}\left(b_{x}-a_{x}\right)$ and $Z_{a x}=z_{0}\left(a_{x}\right)\left(b_{x}-a_{x}\right)$, $Z_{b x}=z_{0}\left(b_{x}\right)\left(b_{x}-a_{x}\right)$.

The above equation may be solved graphically and the set of eigenvalues $\left\{k_{\mu}\right\}$ may be read from the graph.

Substituting the obtained above results into Eq. (27) one obtains the general solution and the set of eigenfunctions $\left\{X_{\mu}(x)\right\}$ in $x$-direction,

$$
\begin{aligned}
X(x) & =\sum_{\mu} C_{2 \mu} X_{\mu}(x) \\
& =\sum_{\mu} C_{2 \mu}\left[\left(k_{\mu} / z_{0}\left(a_{x}\right)\right) \cos \left(k_{\mu} x\right)+\sin \left(k_{\mu} x\right)\right],
\end{aligned}
$$

where $X_{\mu}(x)=[\ldots]$.

By analogy, the general solution of Eq. (10) and the set eigenfunctions $\left\{X_{\iota}(x)\right\}$ in $y$-direction take the form

$$
\begin{aligned}
Y(y) & =\sum_{\iota} C_{2 \iota} Y_{\iota}(y) \\
& =\sum_{\iota} C_{2 \iota}\left[\left(k_{\iota} / z_{0}\left(a_{y}\right)\right) \cos \left(k_{\iota} y\right)+\sin \left(k_{\iota} y\right)\right],
\end{aligned}
$$

where $k_{\iota}=k_{y}$ and $Y_{\iota}(y)=[\ldots]$.

Now, one solves Eq. (11) in the $z$-direction, with boundary conditions Eqs. (25) and (26). The general solution takes the form, cf. Eq. (27),

$$
Z(z)=D_{1} \cos \left(k_{\nu} z\right)+D_{2} \sin \left(k_{\nu} z\right)
$$

where $k_{\nu}=k_{z}$.

Substituting Eq. (31) into Eqs. (25) and (26) gives $D_{2}=0$. Next, the set of eigenvalues $\left\{k_{\nu}\right\}$ ought to be found. They are solutions of the eigenvalues equation which can be obtained from Eq. (29). For this purpose one takes: $k_{\mu} \equiv k_{\nu}, a_{x} \equiv a_{z}, b_{x} \equiv b_{z}$, $z_{0}\left(a_{x}\right) \equiv z_{0}\left(a_{z}\right)=0, z_{0}\left(b_{x}\right) \equiv z_{0}\left(b_{z}\right)$, hence instead of Eq. (28) it is

$$
\tan \left(w_{z}\right)=Z_{b z} / w_{z}
$$

where $w_{z}=k_{\nu}\left(b_{z}-a_{z}\right)$ and $Z_{b z}=z_{0}\left(b_{z}\right)\left(b_{z}-a_{z}\right)$.

Substituting the results obtained above into Eq. (31) leads to the set of eigenfunctions $\left\{Z_{\nu}(z)\right\}$,

$$
Z(z)=\sum_{\nu} D_{1 \nu} Z_{\nu}(z)=\sum_{\nu} D_{1 \nu} \cos \left(k_{\nu} z\right),
$$

where $Z_{\nu}(z)=\cos \left(k_{\nu} z\right)$.

In the end, the $\mu \iota \nu$-eigenfunctions ( $\mu \iota \nu$-modes) are expressed with the product

$$
U_{\mu \iota \nu}(\mathbf{x})=X_{\mu}(x) Y_{\iota}(y) Z_{\nu}(z) .
$$

As it can be seen, all solutions of the 3D indoor acoustic problems may be achieved through the Fourier method.

\section{Forced acoustic vibrations}

The differential equation of these vibrations is given by Eq. (4). Let initial conditions be zeros. For problem formulated this way, the solution of it is given by the equation $u(\mathbf{x}, t)=u_{1}(\mathbf{x}, t)+u_{2}(\mathbf{x}, t)$, where $u_{1}(\mathbf{x}, t)$ are the free vibrations, $u_{2}(\mathbf{x}, t)$ are the forced vibrations. In the following, just forced vibrations ought to be found; for simplicity of their notation $u_{2}(\mathbf{x}, t)=u(\mathbf{x}, t)$.

The periodic vibrations have a practical meaning and they, in turn, can be described by means of harmonic vibrations. Acoustic vibrations are excited through $f(\mathbf{x}, t)$, in Eq. (4), i.e., the point acoustic source. It is assumed that the source (a pulsating little sphere) vibrates with an angular frequency $\omega_{f}$. At the beginning, let

$$
f(\mathbf{x}, t)=F(\mathbf{x}) e^{i \omega_{f} t} .
$$

The forced acoustic vibrations in the room are assumed in the similar form

$$
u(\mathbf{x}, t)=U(\mathbf{x}) e^{i \omega_{f} t} .
$$

Substituting Eqs. (35) and (36) in Eq. (4), one obtains the inhomogeneous equation of excited acoustic vibrations in the steady state (Helmholtz inhomogeneous equation)

$$
D^{2} U(\mathbf{x})+k_{f}^{2} U(\mathbf{x})=F(\mathbf{x})
$$

where $k_{f}=\omega_{f} / c$.

An acoustic source in $3 \mathrm{D}$ is represented by the function $F(\mathbf{x})$ in Eq. (35). In mathematical interpretation, the $F(\mathbf{x})$ constitutes the solution of the radial part of the Bessel's differential equation in spherical coordinates (KoRN, KorN, 1968; MCLACHLAN, 
1964; Evans, 2002). Here, the 0-order, spherical Hankel function of the second kind $h_{0}^{(2)}\left(k_{f} r\right)$ plays the major role. This is because it describes the outward propagating spherical wave.

The solution of Eq. (37) is now formulated as some sum of eigenfunctions. In a similar manner, the source function $F(\mathbf{x})$ is represented, then

$$
\begin{aligned}
U(\mathbf{x}) & =\sum_{\mu \iota \nu} a_{\mu \iota \nu} U_{\mu \iota \nu}(\mathbf{x}) \\
& =\sum_{\mu \iota \nu} a_{\mu \iota \nu} X_{\mu}(x) Y_{\iota}(y) Z_{\nu}(z), \\
F(\mathbf{x}) & =\sum_{\mu \iota \nu} b_{\mu \iota \nu} U_{\mu \iota \nu}(\mathbf{x}) \\
& =\sum_{\mu \iota \nu} b_{\mu \iota \nu} X_{\mu}(x) Y_{\iota}(y) Z_{\nu}(z),
\end{aligned}
$$

where $\left\{a_{\mu \iota \nu}\right\},\left\{b_{\mu \iota \nu}\right\}$ are sets of some constants.

Since the function of $F(\mathbf{x})$ is known in advance, then constants $b_{\mu \iota \nu}$ are first calculated. The Eq. (39) is the expansion of $F(\mathbf{x})$ in the triple Fourier series. In such case, constants $b_{\mu \iota \nu}$ are given by the formula

$$
\begin{aligned}
b_{\mu \iota \nu}= & \left(1 / \beta_{\mu \iota \nu}\right) \int_{a_{x}}^{b_{x}} \int_{a_{y}}^{b_{y}} \int_{a_{z}}^{b_{z}} \\
& \cdot F(\mathbf{x}) X_{\mu}(x) Y_{\iota}(y) Z_{\nu}(y) \mathrm{d} x \mathrm{~d} y \mathrm{~d} z,
\end{aligned}
$$

where

$$
\begin{aligned}
\beta_{\mu \iota \nu} & =\beta_{\mu} \beta_{\iota} \beta_{\nu} \\
& =\int_{a_{x}}^{b_{x}} X_{\mu}^{2}(x) \mathrm{d} x \int_{a_{y}}^{b_{y}} Y_{\iota}^{2}(y) \mathrm{d} y \int_{a_{z}}^{b_{z}} Z_{\nu}^{2}(z) \mathrm{d} z .
\end{aligned}
$$

Now, constants $a_{\mu \iota \nu}$ should be computed. To achieve this, Eqs. (38) and (39) are substituted into Eq. (37), hence

$\sum_{\mu \iota \nu} a_{\mu \iota \nu}\left(D^{2} U(\mathbf{x})+k_{f}^{2} U_{\mu \iota \nu}(\mathbf{x})\right)=\sum_{\mu \iota \nu} b_{\mu \iota \nu} U_{\mu \iota \nu}(\mathbf{x})$.

Since

$$
D^{2} U_{\mu \iota \nu}(\mathbf{x})+k_{\mu \iota \nu}^{2} U_{\mu \iota \nu}(\mathbf{x})=0,
$$

hence $D^{2} U_{\mu \iota \nu}(\mathbf{x})=-k_{\mu \iota \nu}^{2} U_{\mu \iota \nu}(\mathbf{x})$ and instead of Eq. (42) one has

$$
\sum_{\mu \iota \nu} a_{\mu \iota \nu}\left(k_{f}^{2}-k_{\mu \iota \nu}^{2}\right) U_{\mu \iota \nu}(\mathbf{x})=\sum_{\mu \iota \nu} b_{\mu \iota \nu} U_{\mu \iota \nu}(\mathbf{x}),
$$

where $k_{\mu \iota \nu}^{2}=k_{\mu}^{2}+k_{\iota}^{2}+k_{\nu}^{2}$.

Comparing the coefficients at the same separate modes $U_{\mu \iota \nu}(\mathbf{x})$ one obtains

$$
a_{\mu \iota \nu}=\frac{b_{\mu \iota \nu}}{\left(k_{f}^{2}-k_{\mu \iota \nu}^{2}\right)} .
$$

Substituting Eq. (45) into Eq. (38) gives

$$
\begin{aligned}
U(\mathbf{x}) & =\sum_{\mu \iota \nu} a_{\mu \iota \nu} U_{\mu \iota \nu}(\mathbf{x}) \\
& =\sum_{\mu \iota \nu} \frac{b_{\mu \iota \nu}}{\left(k_{f}^{2}-k_{\mu \iota \nu}^{2}\right)} X_{\mu}(x) Y_{\iota}(y) Z_{\nu}(z)
\end{aligned}
$$

Finally, forced acoustical vibrations are described by Eq. (36).

In practice, instead of the acoustic potential, the acoustic pressure and secondary acoustic quantities play a major role. First of all, the amplitude of the acoustic pressure by Eq. (14) is calculated and consequently, the value of the sound pressure level $L(\mathbf{x})[\mathrm{dB}]$ is given by

$$
L(\mathbf{x})=20 \log \left|p(\mathbf{x}) / p_{0}\right|,
$$

where $p(\mathbf{x})$ is the acoustics pressure and $p_{0}=2 \cdot 10^{-5} \mathrm{~Pa}$.

To notice quantitative change of the $L(\mathbf{x})$ in a whole acoustic room, the mean value of it ought to be calculated based on the equation

$$
L_{m}=1 / n_{i} \sum_{i} L\left(\mathbf{x}_{i}\right)
$$

where $i=1,2, \ldots, n_{i}$ number of calculated points inside the acoustic room.

\section{Numerical calculations}

In this section, numerical calculations are performed assuming the following values: absorption coefficients: $\{\alpha\}=\{0$, step $0.1,1\}$ and frequency: $f=$ $250 \mathrm{~Hz}$. Numerical details are presented for chosen values of absorption coefficients, i.e. $\{\alpha\}=\{0.1,0.5,0.9\}$.

The following global values and symbols are assumed: $\rho=1.205 \mathrm{~kg} / \mathrm{m}^{3}, c=344 \mathrm{~m} / \mathrm{s},\left\{a_{x}, b_{x}\right\}=$ $\{0,5\} \mathrm{m},\left\{a_{y}, b_{y}\right\}=\{0,5\} \mathrm{m},\left\{a_{z}, b_{z}\right\}=\{0,2.5\} \mathrm{m}$, the point forced source is placed at the point $\mathbf{x}_{0}=\left\{x_{0}, y_{0}, z_{0}\right\}=\{2.5,2.5,1.25\} \mathrm{m}$. Furthermore, $z_{0}\left(a_{x}\right) \equiv z_{0}\left(b_{x}\right) \equiv z_{0}\left(b_{y}\right)=Z$, since it is the most frequent assumption made in acoustics, where e.g. $z_{0}\left(a_{x}\right)$ is $z_{0}(x)$ on the edge $a_{x}$ and so on. However, $z_{0}\left(a_{y}\right)=0$ as a result of the Neumann boundary condition. Hence $Z_{a x}=Z_{b x}=Z\left(b_{x}-a_{x}\right)=Z_{x}$.

In the $x$-direction, instead of Eq. (28), the following eigenvalue equation is obtained:

$$
\tan \left(w_{x}\right)=\frac{2 w_{x} Z_{x}}{w_{x}^{2}-Z_{x}^{2}} .
$$

The same equation as Eq. (49) for the $y$-direction is derived; in this case $w_{x}$ ought to be replaced by $w_{y}$ and $Z_{x}$ is replaced by $Z_{y}$.

In the same way, in the $z$-direction, instead of Eq. (32) the following eigenvalue equation is obtained:

$$
\tan \left(w_{z}\right)=Z_{z} / w_{z}
$$

where $Z_{z}=Z\left(b_{z}-a_{z}\right)$. 


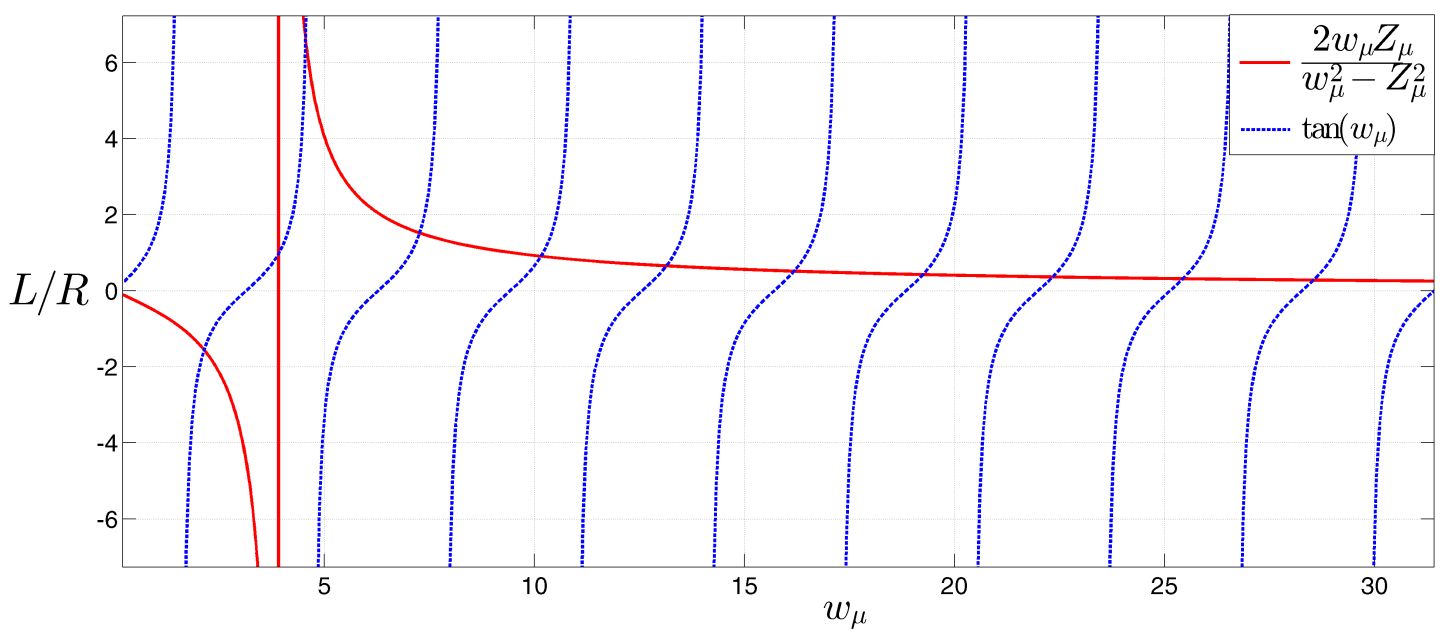

Fig. 2. Graphical solution of Eq. (49), $\alpha=0.5$.

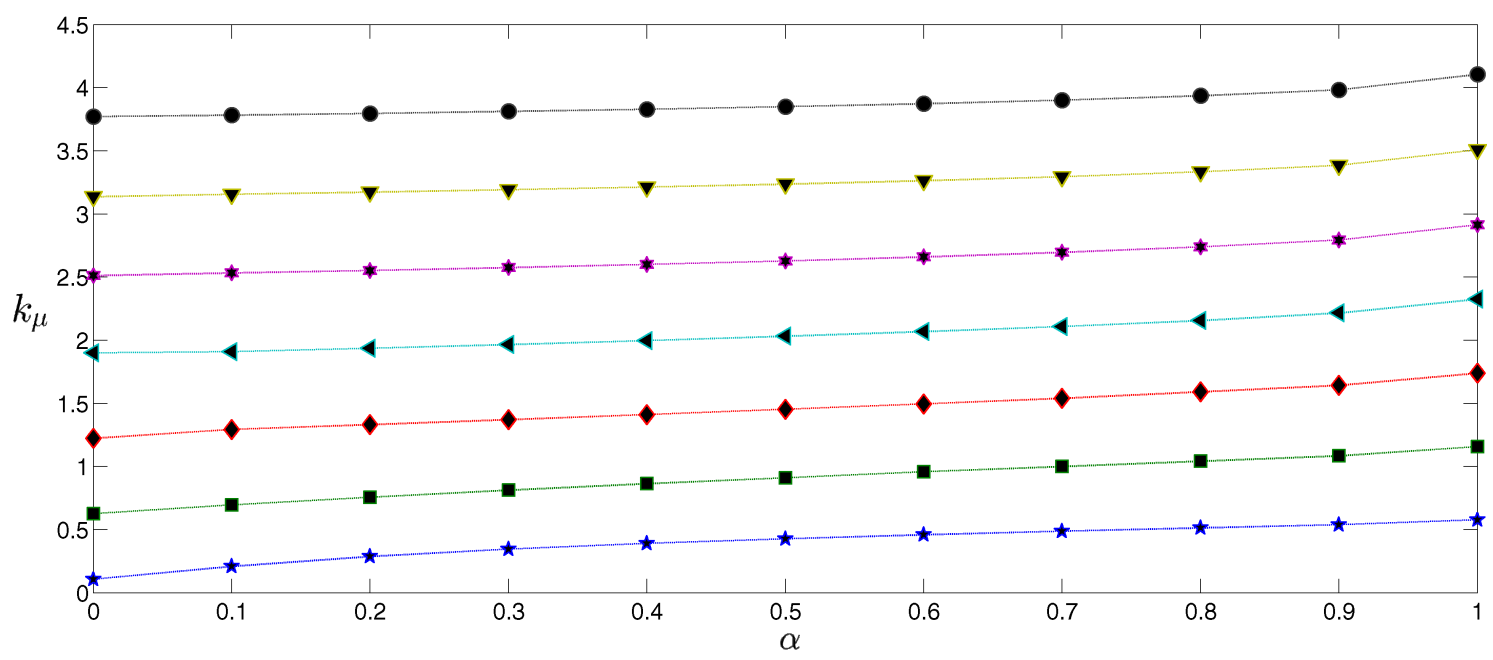

Fig. 3. Graph of $\left\{k_{\mu}\right\}$ versus $\alpha$; e.g. stars $(\leftrightarrow)$ mean first values of $k_{\mu}$ for different values of $\alpha$.

At the first step, sets of eigenvalues $\left\{w_{x}\right\} /\left\{w_{y}\right\}$ and $\left\{w_{z}\right\}$ are calculated in all coordinate directions. So, in $x$-/y-direction, sets $\left\{k_{\mu}\right\} /\left\{k_{\iota}\right\}$ and $\left\{k_{\nu}\right\}$ can be calculated based on Eq. (49) and Eq. (50), respectively.

The eigenvalues equation, Eq. (49), may be solved in many ways (AlquRAN, Al-Khaled, 2010), but in this work it is solved in the graphical manner (PEIRCE, 2014; MeZIANI, 2016). It is done by finding the points of intersection of graphs of the left hand side (LHS/L) and right hand side (RHS/R) of Eq. (49). For example, the result for $\alpha=0.5$ is depicted in Fig. 2, where dashed lines and solid lines on the graphs mean $L$ and $R$, respectively. The results for other values of $\alpha$ are calculated in the same way. To make the paper shorter, the results are presented in detail in $x$-direction, i.e. $\left\{w_{x}\right\}$ and $\left\{k_{\mu}\right\}$, but in $y$-direction $\left\{w_{y}\right\}$ and $\left\{k_{\iota}\right\}$ are written by analogy.

It was assumed that seven values of $w_{\mu}$ ought to be used in order to obtain correct results, hence the $w_{\mu}$-axis on the graphs is limited to the range $w_{\mu} \in$
$[0,30]$. After retrieving values $\left\{w_{\mu}\right\}$ from Fig. 2, the set of $\left\{k_{\mu}\right\}=\left\{k_{\mu}(\alpha)\right\}$ is calculated. The results of $\left\{k_{\mu}\right\}$ for all values of $\{\alpha\}=\{0.1$, step $0.1,0.9\}$ are depicted in Fig. 3, where one marker type represents successive values of $k_{\mu}$ for different values of $\alpha$ (stars represent first values of $k_{\mu}$, rectangles stand for second ones, etc.). Note that the set of $\left\{k_{\iota}\right\}$ is the same as the set of $\left\{k_{\mu}\right\}$.

Next, in quite the same way as above, the solution of Eq. (50) in $z$-direction is derived. First the set $\left\{w_{\mu}\right\}$ and next the set $\left\{k_{\nu}\right\}$ are calculated; the results for $\alpha=0.5$ are presented in Fig. 4 and Fig. 5, respectively.

In the next step, sets of eigenfunctions are calculated. So, in $x$-/y-directions, basing on Eqs. (29) and (30), sets $\left\{X_{\mu}(x)\right\} /\left\{Y_{\iota}(y)\right\}$ are calculated respectively. But in the $z$-direction, basing on Eq. (33), the set $\left\{Z_{\nu}(z)\right\}$ is calculated. To use the Fourier method, the values of orthogonality of the $\left\{X_{\mu}(x)\right\},\left\{Y_{\nu}(y)\right\}$ and $\left\{Z_{\nu}(z)\right\}$, through Eq. (41), are calculated. As a re- 


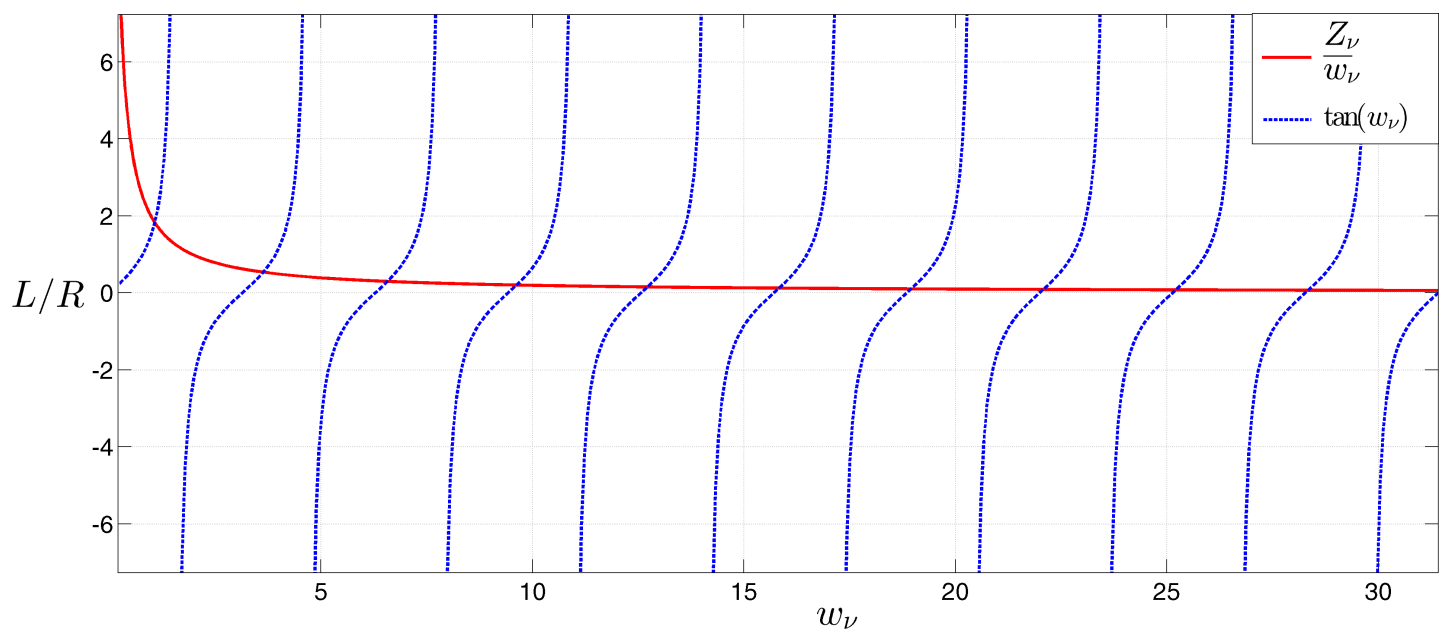

Fig. 4. Graphical solution of Eq. (50), $\alpha=0.5$.

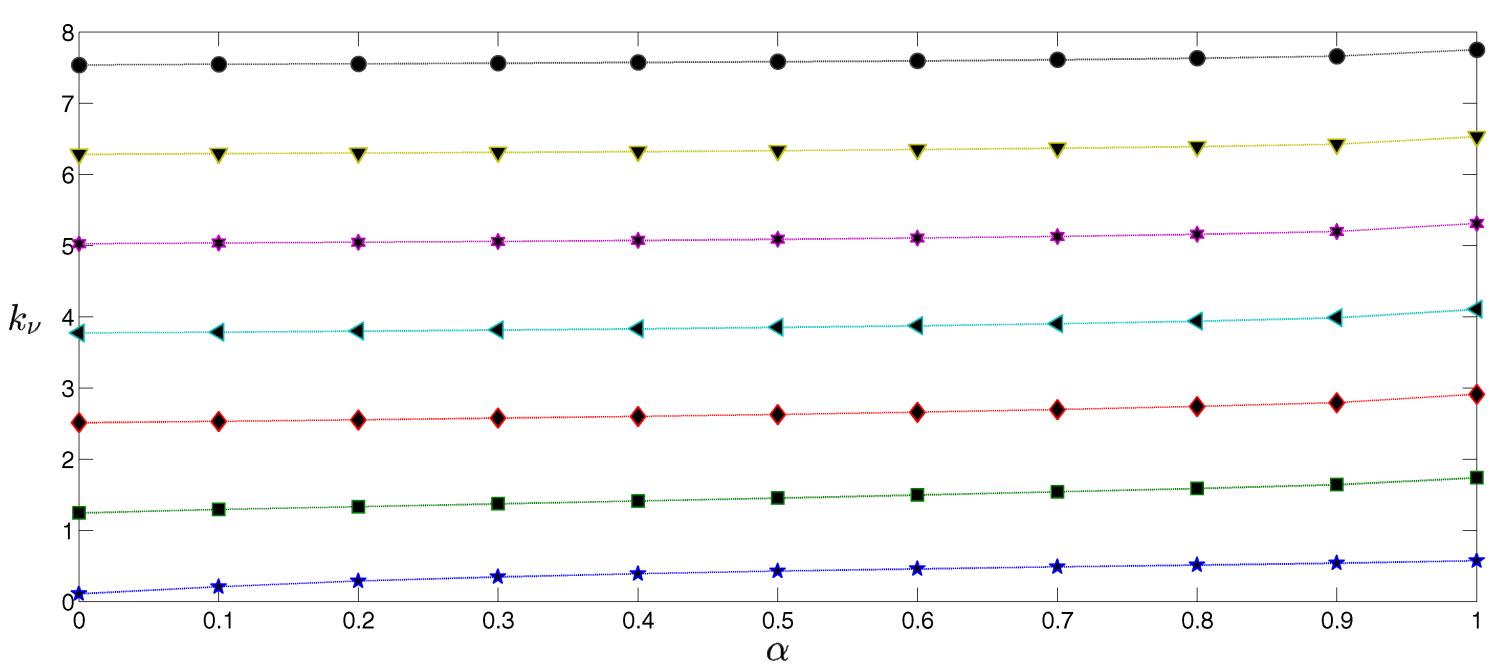

Fig. 5. Graph of $\left\{k_{\nu}\right\}$ versus $\alpha$; e.g. stars $(\leftrightarrow)$ mean first values of $k_{\nu}$ for different values of $\alpha$.

sult, sets $\left\{\beta_{\mu}\right\},\left\{\beta_{\iota}\right\}$, and $\left\{\beta_{\nu}\right\}$ are obtained and they are presented in Tables 1-3.

Table 1. Values of $\left\{\beta_{\mu}\right\} /\left\{\beta_{\iota}\right\}$ and $\left\{\beta_{\nu}\right\}, \alpha=0.1$.

\begin{tabular}{|c|c|c|c|c|c|c|c|}
\hline$\left\{\beta_{\mu}\right\} /\left\{\beta_{\iota}\right\}$ & 6.09 & 2.82 & 2.59 & 2.54 & 2.52 & 2.51 & 2.51 \\
\hline$\left\{\beta_{\nu}\right\}$ & 2.28 & 1.28 & 1.25 & 1.25 & 1.25 & 1.25 & 1.25 \\
\hline
\end{tabular}

Table 2. Values of $\left\{\beta_{\mu}\right\} /\left\{\beta_{\iota}\right\}$ and $\left\{\beta_{\nu}\right\}, \alpha=0.5$.

\begin{tabular}{|c|c|c|c|c|c|c|c|}
\hline$\left\{\beta_{\mu}\right\} /\left\{\beta_{\iota}\right\}$ & 15.2 & 5.29 & 3.59 & 3.06 & 2.83 & 2.72 & 2.65 \\
\hline$\left\{\beta_{\nu}\right\}$ & 1.74 & 1.39 & 1.30 & 1.27 & 1.26 & 1.25 & 1.25 \\
\hline
\end{tabular}

Table 3. Values of $\left\{\beta_{\mu}\right\} /\left\{\beta_{\iota}\right\}$ and $\left\{\beta_{\nu}\right\}, \alpha=0.9$.

\begin{tabular}{|c|c|c|c|c|c|c|c|}
\hline$\left\{\beta_{\mu}\right\} /\left\{\beta_{\iota}\right\}$ & 59.1 & 16.5 & 8.59 & 5.84 & 4.60 & 3.93 & 3.53 \\
\hline$\left\{\beta_{\nu}\right\}$ & 1.45 & 1.39 & 1.33 & 1.30 & 1.28 & 1.27 & 1.26 \\
\hline
\end{tabular}

The information about values of $\left\{\beta_{\mu \iota \nu}\right\}$ permits to calculate $\left\{b_{\mu \iota \nu}\right\}$ by Eq. (40) and $\left\{a_{\mu \iota \nu}\right\}$ by Eq. (45), respectively. In the following it is assumed that $F(x)=$ $A h_{0}^{(2)}\left(k_{f} r\right)$ and, to make the final results real, an intensity of the source $A=5.51 \cdot 10^{-3}$ is assumed. To omit the singularity of $h_{0}^{(2)}\left(k_{f} r\right)$ at $r=0$, some space around the source is omitted; this is the cube space with dimensions $0.2 \times 0.2 \times 0.2 \mathrm{~m}$. Finally, the sound pressure level $L(\mathbf{x})$, through Eq. (47), is calculated.

Since the depiction of function in $3 \mathrm{D}$ space is difficult, the results are presented in selected, characteristic surfaces or lines. First, the pictures of $L(\mathbf{x})$ are shown in Figs. 6-8. They are calculated only for $\alpha=0.5$ in planes which are parallel to the $\pi_{x y}$ Cartesian plane, namely $z=b_{z} / 5, z=b_{z} / 2$, and $z=4 / 5 b_{z}$, respectively.

Next, distributions of $L(\mathbf{x})$, in particular of $\alpha=$ $\{0.1,0.3,0.5,0.7,0.9\}$ and along particular lines are calculated. For fixed $y=2.5, z=1.25$, and $x \in(0 ; 5)$ the results are presented in Fig. 9. For fixed $x=2.5$, $y=2.5$, and $z \in(0 ; 2.5)$ the results are presented in Fig. 10. 


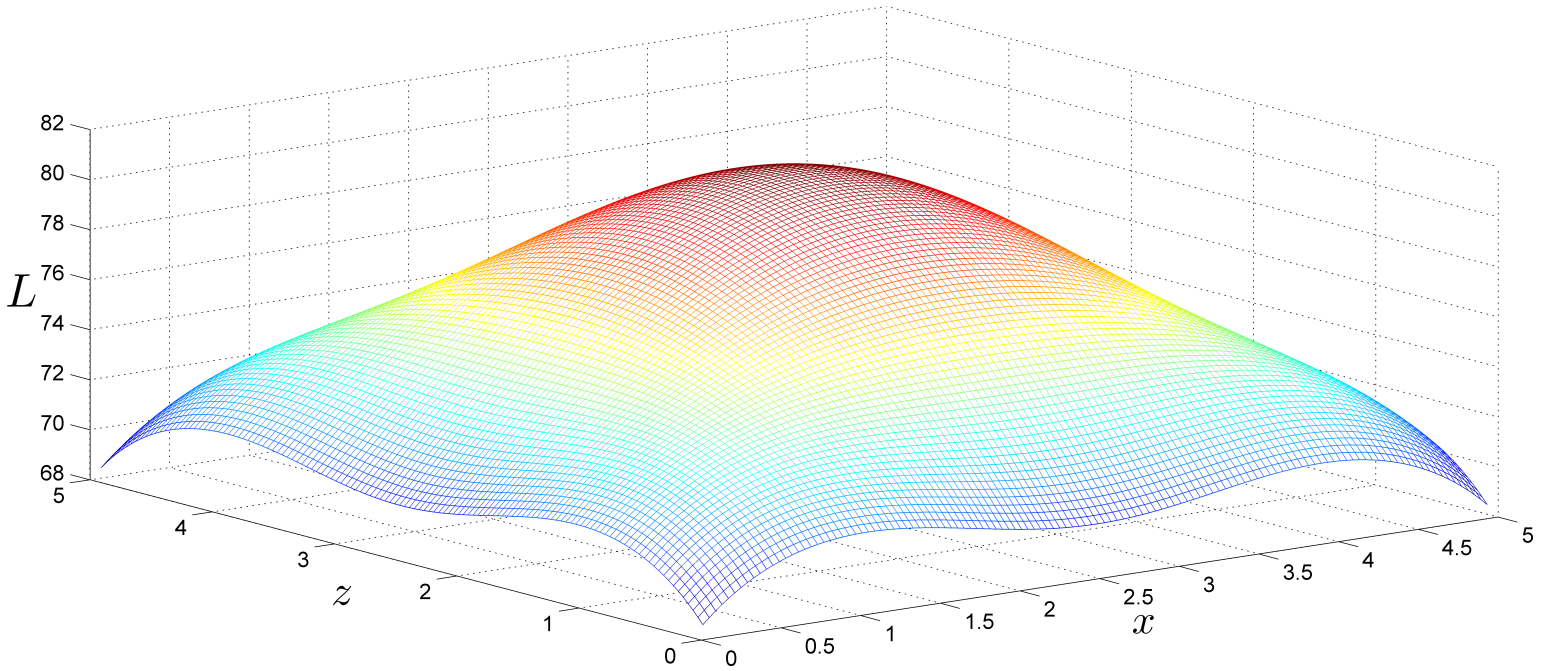

Fig. 6. Level $L(\mathbf{x})$ in the cross-section for $z=b_{z} / 5$.

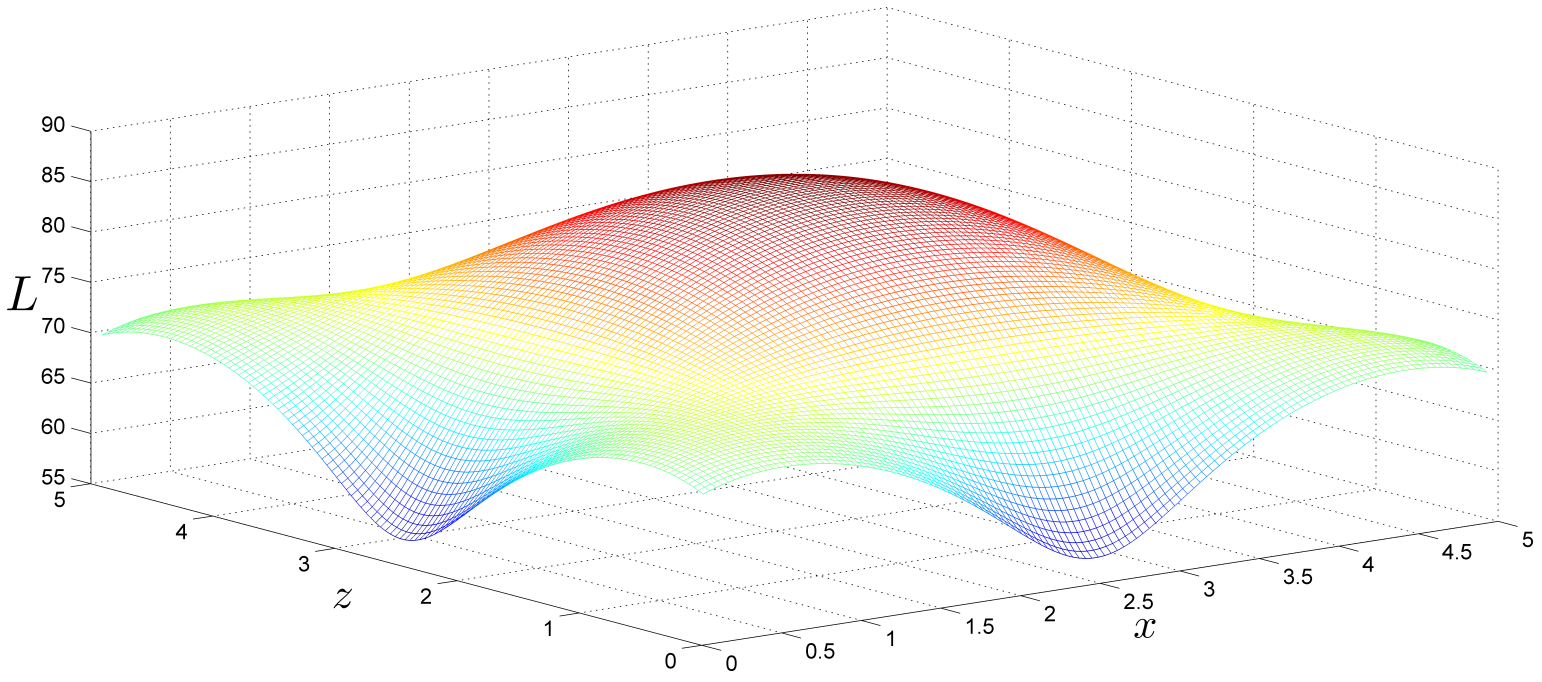

Fig. 7. Level $L(\mathbf{x})$ in the cross-section for $z=b_{z} / 2$.

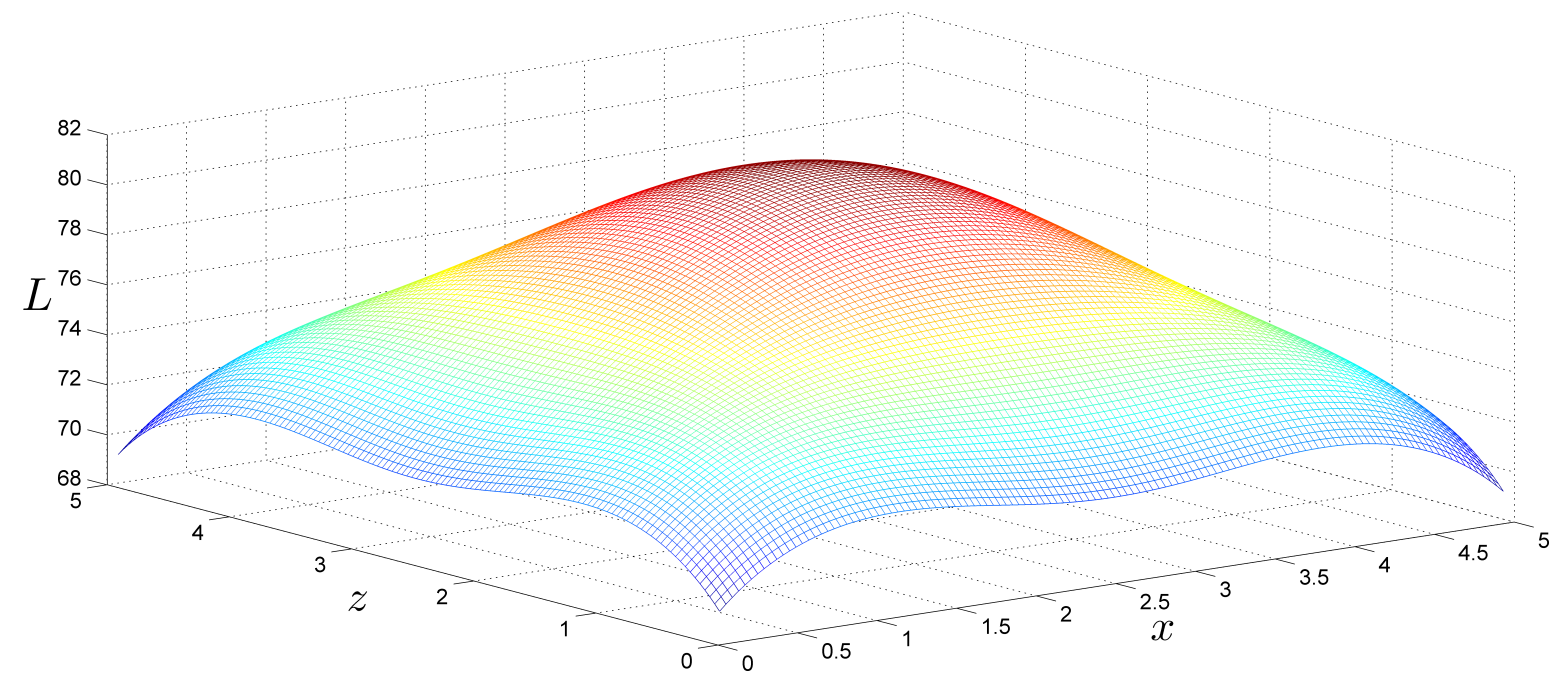

Fig. 8. Level $L(\mathbf{x})$ in the cross-section for $z=4 / 5 b_{z}$. 


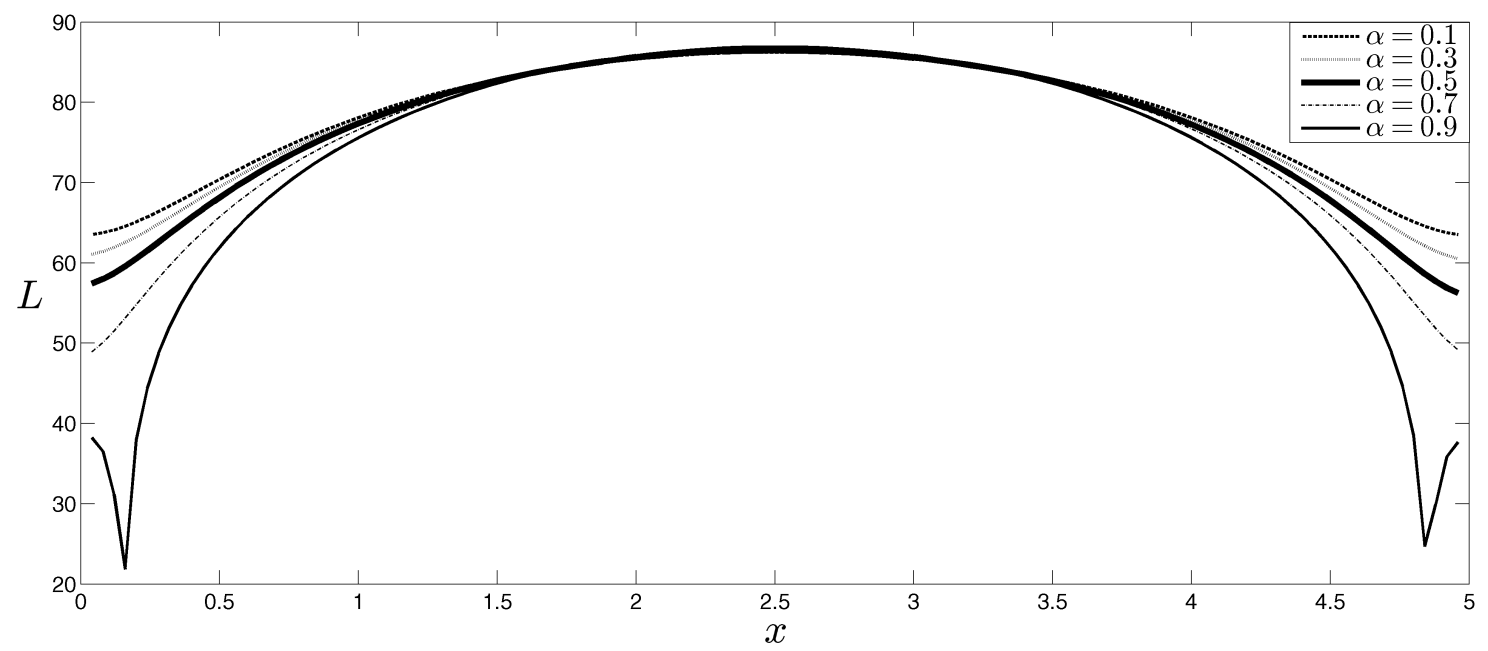

Fig. 9. Cross-sections of $L(\mathbf{x})$ for fixed $y=2.5$ and $z=1.25$; for $\alpha=[0.1,0.3,0.5,0.7,0.9]$ the set of $L_{m}=[78.4665,77.9663,77.2491,76.0666,73.2667]$.

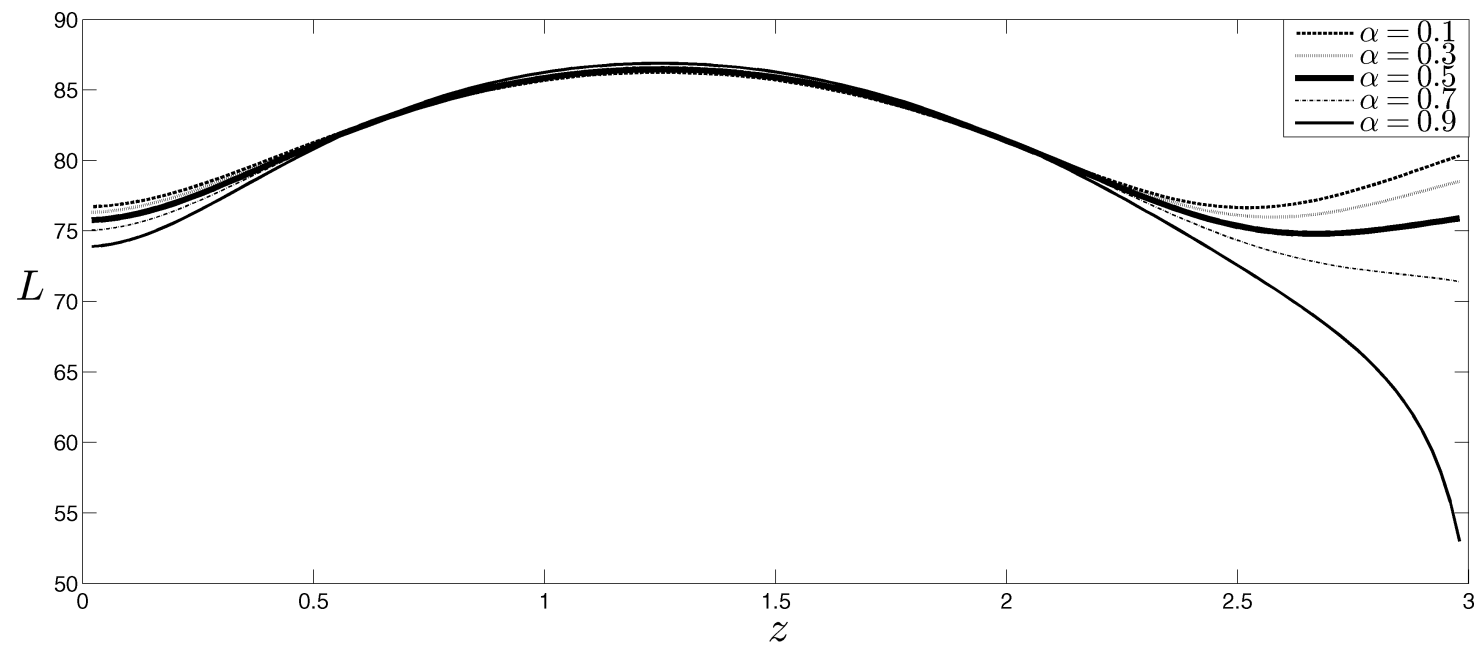

Fig. 10. Cross-sections of $L(\mathbf{x})$ for fixed $x=2.5$ and $y=2.5$; for $\alpha=[0.1,0.3,0.5,0.7,0.9]$ the set of $L_{m}=[97.9261,97.6395,97.2225,96.6212,95.1277]$.

\section{Conclusions}

The exact solution of the $3 \mathrm{D}$ acoustic problem with arbitrary but uniform impedances imposed on separate walls by the Fourier method was achieved. Small errors were introduced to the solution through approximate solutions of eigenvalue equations. The theoretical considerations were verified by numerical simulations. One rigid wall (the floor) and the same impedance rest walls were assumed.

The acoustic field was presented by the distribution of the sound pressure level and its mean value. Calculations were performed for all values of the absorption coefficient $\alpha(\mathbf{x})$, namely, $\alpha(\mathbf{x})=\{0.1$, step $0.1,0.9\}$ and for annular frequency $f=250 \mathrm{~Hz}$. The results are presented in some cross-sections of the room. Based on theoretical considerations and numerical simulations some conclusions can be drawn.
1. It is possible to obtain the solution of the acoustic boundary problem with impedance boundary conditions by Fourier method. In the 3D space, this problem is described by the combination of three 1D Sturm-Liouville appropriate boundary problems in separate directions. This way the paper partially fills the gap of the practical acoustic boundary problem.

2. A certain restriction appears, namely, the impedance condition must be uniform along the surface of particular walls coinciding with appropriate coordinate axes. However, there are no limits to the impedance values on opposite walls.

3. Although the Fourier method belongs to the exact group of methods, due to the lack of exact solutions of eigenvalues equations, it loses this feature. However, the error introduced by the graphical solution of these equations seems to be small. 
4. Quantitative results of the Fourier method can be a point of reference to the results of numerical methods.

\section{Acknowledgments}

Scientific work was financed by the Ministry of Science and Higher Education from the budget for science in years 2015-2017 as a research project.

\section{References}

1. Alquran M., Al-Khaled K. (2010), Approximations of Sturm-Liouville eigenvalues using sin-Galerkin and differential transform methods, Applications and Applied Mathematics, 5, 128-147.

2. Bistafa R.S., Morrissey J.W. (2003), Numerical solution of the acoustic eigenvalue equation in the rectangular room with arbitrary (uniform) wall impedances, Journal of Sound and Vibration, 263, 205-218.

3. Blackstock D.T. (2000), Fundamentals of physical acoustics, Wiley-Interscience, New York.

4. Bowles R. (2007), MATHM242 Lecture Notes - first half, University College London, London.

5. BRAŃsKi A. (2013), Numerical methods to the solution of boundary problems, classification and survey [in Polish], Rzeszow University of Technology Press, Rzeszow. ISBN 978-83-7199-792-1.

6. Brański A., Borkowska D. (2015), Effectiveness of nonsingular solutions of the boundary problems based on Trefftz methods, Engineering Analysis with Boundary Elements, 59, 97-104.

7. Brański A., Borkowski M., Borkowska D. (2012), A comparison of boundary methods based on inverse variational formulation, Engineering Analysis with Boundary Elements, 36, 505-510.

8. Chen W., Zhang J.Y., Fu Z.J. (2014), Singular boundary method for modified Helmholtz equations, Engineering Analysis with Boundary Elements, 44, 112119.

9. Dance S., Van Buuren G. (2013), Effects of damping on the low-frequency acoustics of listening rooms based on an analytical model, Journal of Sound and Vibration, 332, 6891-6904.

10. Dautray R., Lions J.L. (2000), Mathematical analysis and numerical methods for science and technology, Springer, Berlin.

11. Ducourneaua J., Planeaub V. (2003), The average absorption coefficient for enclosed spaces with nonuniformly distributed absorption, Applied Acoustics, 64, 845-862.

12. Evans L.C. (2002), Partial differential equations [in Polish], WN PWN, Warszawa.

13. Fasshauer G. (2011), MATH 461: Fourier Series and Boundary Value Problems. Chapter V: Sturm-Liouville Eigenvalue Problems, Department of Applied Mathematics, Illinois Institute of Technology, Fall.
14. Fu Z.J., Chen W., Gu Y. (2014), Burton-Miller-type singular boundary method for acoustic radiation and scattering, Journal of Sound and Vibration, 333, 37763793.

15. Gerai M. (1993), Measurement of the sound absorption coeffiients in situ: the reflection method using periodic pseudo-random sequences of maximum length, Applied Acoustics, 39, 119-139.

16. International Organization for Standardization ISO 354 (2003), Acoustics - measurement of sound absorption in a reverberation room.

17. International Organization for Standardization ISO 10354-1 (1996), Acoustics - determination of sound absorption coefficient and impedance in impedance tube. Part 1: method using standing wave ratio.

18. Johnson R.S. (2006), An introduction to SturmLiouville theory, School of Mathematics \& Statistics - University of Newcastle upon Tyne.

19. Kashdan E. (2017), ACM 30020 Advanced Mathematical Methods, Sturm-Liouville Problem (SLP), http://mathsci.ucd.ie/ ekashdan/page2/SLP (accessed March 17, 2017).

20. Korany N., Blauert J., Abdel Alim O. (2001), Acoustic simulation of rooms with boundaries of partially specular reflectivity, Applied Acoustic, 62, 875887.

21. Korn G.A., Korn T.M. (1968), Mathematical handbook for scientists and engineers, McGraw-Hill Book Company, New York.

22. Kuttruff H. (2000), Room acoustics, Fundamentals of Physical Acoustics, Wiley-Interscience, New York.

23. Lehmann E., Johansson A. (2008), Prediction of energy decay in room impulse responses simulated with an image-source model, Journal of the Acoustical Society of America, 124, 269-277.

24. Lin J., Chen W., Chen C.S. (2014), Numerical treatment of acoustic problems with boundary singularities by the singular boundary method, Journal of Sound and Vibration, 333, 3177-3188.

25. Lopez J., Carnicero D., Ferrando N., EscolaNO J. (2013), Parallelization of the finite-difference time-domain method for room acoustics modelling based on CUDA, Mathematical and Computer Modelling, 57, 1822-1831.

26. Luizard P., Polack J.P., Katz B. (2014), Sound energy decay in coupled spaces using a parametric analytical solution of a diffusion equation, Journal of the Acoustical Society of America, 135, 2765-2776.

27. MCLaChLAN N.W. (1964), Bessel functions for engineers [in Polish], PWN, Warszawa.

28. Meissner M. (2009a), Computer modelling of coupled spaces: variations of eigenmodes frequency due to a change in coupling area, Archives of Acoustics, 34, $157-168$.

29. Meissner M. (2009b), Spectral characteristics and localization of modes in acoustically coupled enclosures, Acta Acustica united with Acustica, 95, 300-305. 
30. MeIssner M. (2010), Simulation of acoustical properties of coupled rooms using numerical technique based on modal expansion, Acta Physica Polonica A, 118, 123-127.

31. Meissner M. (2012), Acoustic energy density distribution and sound intensity vector field inside coupled spaces, Journal of the Acoustical Society of America, 132, 228-238.

32. Meissner M. (2013a), Analytical and numerical study of acoustic intensity field in irregularly shaped room, Applied Acoustics, 74, 661-668.

33. MEISsner M. (2013b), Evaluation of decay times from noisy room responses with puretone excitation, Archives of Acoustics, 38, 47-54.

34. Meziani H. (2016), Sturm-Liouville Problems, Generalized Fourier Series. http://www2.fiu.edu/ meziani/ NOTE9.pdf (accessed May 11, 2016).

35. Morse P.M., Ingard K.U. (1987), Theoretical acoustics, Princeton University Press, New Jersey.

36. Naka Y., Oberai A.A., Shinn-Cunningham B.G. (2005), Acoustic eigenvalues of rectangular rooms with arbitrary wall impedances using the interval Newton/generalized bisection method, Journal of the Acoustical Society of America, 118, 3662-3671.
37. Neta B. (2012), Partial differential equations, MA 3132 Lecture notes, Monterey, California 93943.

38. Okuzono T., Otsuru T., Tomiku R., Okamoto N. (2014), A finite-element method using dispersion reduced spline elements for room acoustics simulation, Applied Acoustics, 79, 1-8.

39. PeIRCE A. (2014), Lecture 28: Sturm-Liouville Boundary Value Problems, Introductory lecture notes on Partial Differential Equations. The University of British Columbia, Vancouver.

40. Summers J. (2012), Accounting for delay of energy transfer between coupled rooms in statistical-acoustics models of reverberant-energy decay, Journal of the Acoustical Society of America, 132, 129-134.

41. Takahashi Y., Otsuru T., Tomiku R. (2005), In situ measurements of surface impedance and absorption coefficients of porous materials using two microphones and ambient noise, Applied Acoustics, 66, 845-865.

42. Thompson L.L., Pinsky P.-M. (1995), A Galerkin Least Squares Finite Element Method for the twodimensional Helmholtz equation, International Journal Numerical Methods Engineering, 38, 371-397.

43. Xu B., Sommerfeldt S. (2010), A hybrid modal analysis for enclosed sound fields, Journal of the Acoustical Society of America, 128, 2857-2867. 